Virginia Commonwealth University

vCU Scholars Compass

2016

Children exposed to intimate partner violence: Identifying differential effects of family environment on children's trauma and psychopathology symptoms through regression mixture models

Shelby Elaine McDonald

Virginia Commonwealth University, smcdonald3@vcu.edu

Sunny Shin

Virginia Commonwealth University, sshin@vcu.edu

Rosalie Corona

Virginia Commonwealth University, racorona@vcu.edu

See next page for additional authors

Follow this and additional works at: https://scholarscompass.vcu.edu/socialwork_pubs

Part of the Social Work Commons

(C) 2016 Elsevier Ltd

Downloaded from

https://scholarscompass.vcu.edu/socialwork_pubs/18

This Article is brought to you for free and open access by the School of Social Work at VCU Scholars Compass. It has been accepted for inclusion in Social Work Publications by an authorized administrator of VCU Scholars Compass. For more information, please contact libcompass@vcu.edu. 
Authors

Shelby Elaine McDonald, Sunny Shin, Rosalie Corona, Anna Maternick, Sandra A. Graham-Bermann, Frank R. Ascione, and James Herbert Williams 


\title{
Children Exposed to Intimate Partner Violence: Identifying Differential Effects of Family Environment on Children's Trauma and Psychopathology Symptoms through Regression Mixture Models
}

\author{
Shelby Elaine McDonald, $\mathrm{PhD}^{\mathrm{a}}$, Sunny Shin, PhD, Rosalie Corona, $\mathrm{PhD}^{\mathrm{b}}$, Anna Maternick, \\ MS, Sandra A. Graham-Bermann, PhD $^{\mathrm{C}}$, Frank R. Ascione, PhD, and James Herbert \\ Williams, PhD $^{\mathrm{d}}$ \\ aSchool of Social Work, Virginia Commonwealth University, Academic Learning Commons, 1000 \\ Floyd Avenue, Third Floor, P.O. Box 842027, Richmond, VA 23284-2027 \\ ${ }^{b}$ Department of Psychology, Virginia Commonwealth University, 806 W. Franklin Street, \\ Richmond, VA 23284 \\ 'Department of Psychology, The University of Michigan, 2265 East Hall, 530 Church Street, Ann \\ Arbor, Ml 48109-1043 \\ 'Graduate School of Social Work, University of Denver, Craig Hall, 2148 S. High St., Denver, CO \\ 80208
}

\begin{abstract}
The majority of analytic approaches aimed at understanding the influence of environmental context on children's socioemotional adjustment assume comparable effects of contextual risk and protective factors for all children. Using self-reported data from 289 maternal caregiver-child dyads, we examined the degree to which there are differential effects of severity of intimate partner violence (IPV) exposure, yearly household income, and number of children in the family on posttraumatic stress symptoms (PTS) and psychopathology symptoms (i.e., internalizing and externalizing problems) among school-age children between the ages of 7 to 12 years. A regression mixture model identified three latent classes that were primarily distinguished by differential effects of IPV exposure severity on PTS and psychopathology symptoms: (1) asymptomatic with low sensitivity to environmental factors (66\% of children), (2) maladjusted with moderate sensitivity (24\%), and (3) highly maladjusted with high sensitivity (10\%). Children with mothers who had higher levels of education were more likely to be in the maladjusted with moderate sensitivity group than the asymptomatic with low sensitivity group. Latino children were less likely to be in both maladjusted groups compared to the asymptomatic group. Overall, the
\end{abstract}

sem9883@gmail.com (Corresponding Author).

Publisher's Disclaimer: This is a PDF file of an unedited manuscript that has been accepted for publication. As a service to our customers we are providing this early version of the manuscript. The manuscript will undergo copyediting, typesetting, and review of the resulting proof before it is published in its final citable form. Please note that during the production process errors may be discovered which could affect the content, and all legal disclaimers that apply to the journal pertain.

This paper was presented in part at the Annual Meeting of the Society for Social Work and Research in January 2016 in Washington DC. 
findings suggest differential effects of family environmental factors on PTS and psychopathology symptoms among children exposed to IPV. Implications for research and practice are discussed.

\section{Keywords}

Intimate partner violence; Children; Resilience; Adjustment

\section{Introduction}

In the past several decades, increasing attention has been drawn to childhood exposure to intimate partner violence (IPV) and its disruptive impacts on lifelong health, learning, and behavior (Howell, Barnes, Miller, \& Graham-Bermann, 2015). Experiencing the harms associated with IPV through direct or indirect observation is increasingly being identified as a form of child maltreatment in U.S (Gilbert et al., 2009; MacMillen, Wathen, \& Varcoe, 2013). Data from nationally representative samples suggest that $11 \%$ of U.S. children have been exposed to IPV in the past year and $26 \%$ of children have been exposed to IPV in their lifetime (Hamby, Finkelhor, Turner, \& Ormrod, 2011). A large body of research links childhood IPV exposure to impairment in physical and mental health and behavioral problems across the lifespan (e.g., Chan \& Yeung, 2009; Foster \& Brooks-Gunn, 2009; Gewirtz \& Edleson, 2007; Holt, Buckley, \& Whelan, 2008; Kuelbs, 2009; Wolfe, Crooks, Lee, McIntyre-Smith, \& Jaffe, 2003). Among a variety of deleterious consequences associated with IPV exposure, posttraumatic stress (PTS) and internalizing and externalizing problems are among the most well documented in school-age children (Howell et al., 2015). For example, in a meta-analysis of 60 studies that examined the effects of IPV exposure on PTS and psychopathology, Evans, Davies, and DiLillo (2008) reported a large effect size for PTS ( $d=1.54)$, and moderate effect sizes for internalizing problems $(d=.51)$ and externalizing problems $(d=.49)$.

Prior research has also demonstrated several interconnected and often nested individual and family environmental factors that directly and indirectly affect PTS and psychopathology symptoms among children exposed to IPV. They include gender, ethnicity, the severity of children's exposure to IPV (Graham-Bermann, Gruber, Girz, \& Howell, 2009; Grych, Jouriles, Swank, McDonald, \& Norwood 2000; Howell, Graham-Bermann, Czyz, \& Lilly, 2010; Kilpatrick \& Williams, 1998; Spilsbury et al., 2008; Wolfe, et al., 2003), maternal warmth and mental health (Graham-Bermann \& Levendosky, 1998), family income (Graham-Bermann, DeVoe, Mattis, Lynch, \& Thomas, 2006; Shaw, Keenan, Vondra, Delliquandri, \& Giovannelli, 1997), and number of children in the household (Hoffman, Demo, \& Edwards, 1994; Keenan, Gunthorpe, \& Grace, 2007; Trentacosta et al., 2008). Yet, findings regarding individual and family environmental influences vary among different subgroups of children. For example, Graham-Bermann et al. (2006) reported that mothers' mental health and low self-esteem best predicted PTS symptoms among European American children exposed to IPV whereas, for ethnic minority children, low family income and the severity of children's exposure to IPV were the strongest predictors of PTS symptoms. Additionally, maternal social support was a protective factor for PTS symptoms only for minority children. Furthermore, Skopp, McDonald, Jouriles, and Rosenfield, (2007) found 
that while maternal warmth had no moderating effect on the relationship between IPV and externalizing problems for boys, it demonstrated buffering effects for girls' externalizing problems in that exposure to IPV was positively associated with girls' externalizing problems only in the presence of low levels of maternal warmth. Although more evidence is needed, these findings highlight the importance of attending to heterogeneity in children's responses to IPV exposure and differential effects of IPV exposure on PTS and psychopathology symptoms of victimized children.

Within the field of child development, there is increasing recognition of holistic and ecological models of individual development, which focus on the heterogeneity of child development (Bergman \& Trost, 2006; Bronfenbrenner, 1979, 2005; Van Horn et al., 2009). These frameworks aim to delineate how various subgroups of children differ across multiple developmental characteristics, and assume that the influence of the contextual environment on children differs across children due to varying individual and family characteristics (von Eye \& Bogat, 2006; Van Horn et al., 2009). Person-centered methodologies (e.g., cluster analysis, regression mixture models) are consistent with ecological models of child development in that such approaches advocate for attending to socio-contextual risk and protective factors to explain multivarious interactions occurring in the family and broader social environment (Van Horn et al., 2009). Person-oriented frameworks are particularly relevant to the study of children's exposure to IPV because there is great heterogeneity among this population in terms of their IPV exposure severity and the effects of IPV exposure on developmental outcomes (Edleson, 2004). For example, while Kitzmann, Gaylord, Holt, and Kenny's (2003) meta-analysis reported that $37 \%$ of children who witness or personally experience IPV have developmental outcomes that are better than or as good as children who do not experience violence in the home, Sternberg, Baradaran, Abbott, Lamb, and Guterman's (2006) mega-analysis showed that children who directly witness IPV are 1.9 times more likely to exhibit internalizing problems and 1.5 times more likely to demonstrate externalizing problems than non-IPV exposed peers. Finally, person-centered frameworks are also important in IPV research because subgroups of children exposed to IPV may demonstrate unique profiles of risk and protective factors that are associated with patterns of PTS and psychopathology symptoms.

Using a cluster analysis (a form of person-centered analysis) of multiple indicators of children's functioning, a few studies have examined profiles of psychological and behavioral adjustment among children exposed to IPV (Hughes \& Luke, 1998; Graham-Bermann et al., 2009; Grych et al., 2000; Lang \& Stover, 2008; Spilsbury et al., 2008). This sparse but important body of work suggests that between 20\% (Graham-Bermann et al., 2009) and 75\% (Lang \& Stover, 2008) of community-based samples of children exposed to IPV are characterized by resilient and/or asymptomatic patterns of adjustment. In addition, each study reported two to four subgroups of children characterized by patterns of moderate to severe psychopathology symptoms, and by different risk and protective factors (e.g., gender, maternal education, and severity of violence exposure) that uniquely distinguished between patterns of adjustment (Lang \& Stover, 2008; Spilsbury et al., 2008; Graham-Bermann et al., 2009). Among the maladjusted profiles identified across these studies, there has been empirical evidence for groups of children characterized by internalizing problems only (Grych et al., 2000; Spilsbury et al., 2008), externalizing problems only (Grych et al., 2000), 
and co-morbid internalizing and externalizing problems (Graham-Bermann et al., 2009; Grych et al., 2000).

The statistical technique of cluster analysis, however, is characterized by notable limitations (Bergman \& Magnusson, 1997; Distefano \& Kamphaus; 2006) such as the lack of statistical indices to guide the researcher's choice of a final solution and unstable classification of clusters when missing variables exist. To address these limitations, a recent study employed latent profile analysis, a more flexible model-based clustering approach that derives clusters, or latent profiles, using a probabilistic model based on relationships between observed class indicator variables. Specifically, McDonald, Graham-Bermann, Maternick, Ascione, and Williams (in press) identified three distinct latent profiles of psychosocial adjustment among school age children recruited from community-based IPV services. The majority of children demonstrated "resilient" profiles of functioning (66\% of children) with normative levels of psychosocial symptomology; $28 \%$ percent of children demonstrated borderline clinical levels of externalizing and internalizing symptomology only; and a small portion of the sample ( $6 \%$ of children) were characterized by clinically significant levels of internalizing, externalizing, attention, and social problems, as well as high levels of callous/unemotional traits (McDonald et al., in press). Therefore, both cluster analysis and latent profile analysis suggest heterogeneity of psychosocial adjustment among children exposed to IPV.

There is longstanding recognition that the influence of victimization-specific and family contextual factors such as the severity of children's exposure to IPV, family household income, and family size might be differentially related to PTS and psychopathology symptoms among children exposed to IPV (Gewirtz \& Edleson, 2007). To the best of our knowledge, however, no prior research has attempted to identify if there are subgroups of children who demonstrate differential profiles of risk and protective factors related to PTS and psychopathology symptoms in the context of IPV. Characterizing how individual and family risk and protective factors might differently influence psychosocial adjustment of children exposed to IPV would help build evidence base for future practice and research on children exposed to IPV. Regression mixture models (also known as latent class regression) might be potential methodological solutions to identify subgroups of children who are primarily distinguished by differential effects of IPV exposure on PTS and psychopathology symptoms (Van Horn et al., 2009). Unlike traditional variable-centered regression methods, regression mixture models relax the assumption of population homogeneity to allow for the examination of parameter differences among unobserved subpopulations (Ding, 2006; Van Horn et al., 2009). Therefore, it allows for the examination of different effects of an independent variable on an outcome variable (i.e., moderation) without needing to include an interaction term in the analysis as is often done in variable-centered regression methods (Muthén \& Asparouhov, 2009; Van Horn et al., 2009). Accordingly, regression mixture models offer a comprehensive assessment of differential effects by attending to complex patterns of relationships between multiple predictor and outcome variables that differ in their relationships across subgroups (Van Horn et al., 2009). In contrast to approaches such as latent class and profile analysis, which classify individuals based on relationships between observed class indicators, regression mixture models classify participants into the same latent group based on relationships between predictor and outcome variables. Thus, this approach is particularly well suited to examining associations between developmental 
contexts (e.g., severity of IPV exposure) and children's adjustment when there is evidence to suggest that contextual effects are not consistent across individuals (Van Horn et al., 2009; Wedel \& DeSarbo, 1995).

\section{Current Study}

Exposure to IPV, a form of child maltreatment, has long been linked to psychological maladjustment in childhood. To date, the majority of studies examining how risk and protective factors influence children's adjustment to IPV have assumed that individual and family contextual effects are consistent across individuals, which may contribute to inconsistencies in the direction and strength of relationships between risk/protective factors and children's adjustment to IPV across studies. The current study addresses this notable gap in the literature, using regression mixture models to examine the degree to which there are differential effects of IPV characteristics (i.e., severity of IPV exposure) and family contextual factors (i.e., family income, number of children in the family) on PTS symptoms and externalizing and internalizing problems among school-aged children exposed to IPV. In addition, the moderating effects of child gender, ethnicity, and maternal education on group differences were explored to assess the influence of individual and maternal characteristics on differential responses to IPV exposure.

Given that no prior studies have used a person-centered framework to examine differential effects of family contextual factors as they pertain to children exposed to family violence, no a priori predictions were made regarding the number and type of classes. Instead, the present study was guided by two primary research questions: (1) Are severity of IPV exposure, yearly household income, and number of children in the family differentially related to PTS and psychopathology symptoms for different subgroups of children? If so, what is the optimal number of subgroups of children (i.e., latent classes) that explain differential associations between the IPV and family factors and children's PTS and psychopathology symptoms? (2) Do child gender, ethnicity, and maternal education moderate the subgroup differences in children's adjustments in response to IPV exposure?

\section{Method}

\section{Participant Recruitment and Sample Description}

Data were collected as part of a larger mixed-methods study on children's exposure to IPV and concomitant animal cruelty. The original study acquired human subjects approval from the Institutional Review Board at a Western U.S. university. In accordance with IRB approved protocol, women with children were recruited from 22 community-based domestic violence agencies in Colorado, which provide comprehensive services (e.g., legal, health, mental health, vocational services) as well as crisis-oriented services (e.g., hot lines, temporary shelter). Community-based domestic violence agencies Eligibility criteria for women included: (a) being over the age of 21; (b) experiencing IPV within the past year; (c) having at least one child between the ages of 7-12 in the home; and (d) having had at least one pet in the home within the past year. In the event that a woman had more than one eligible child, she was able to select which child would participate in the study. Designated staff members (including Spanish-English bilingual staff) from each domestic violence 
agency were trained to recruit participants, obtain consent and assent, and administer standardized surveys to eligible maternal caregiver-child dyads. Maternal caregivers and children met separately with the staff member. Approximately $33 \%$ of maternal caregiver interviews were conducted in Spanish; similarly, $30 \%$ of child interviews were conducted in Spanish. Validated Spanish-language versions of the measures were used when possible. For all other measures, English language versions were translated to Spanish by a professional translator using the translation-back-translation procedure.

A total of 291 maternal caregiver-child dyads participated in the study; however, data from two dyads were excluded in the current analyses because these participants were missing data on multiple key variables. Data reflect 289 maternal caregiver-child dyads. Nearly half of the children (47\%) were female and children's mean age was 9.07 years $(S D=1.64)$. The representation of racial and ethnic identities was: 55.2\% Latino or Hispanic, 22.1\% White, $17.9 \%$ more than 1 race, $3.4 \%$ African American or Black, 1.0\% American Indian, and 0.3\% Asian. Maternal caregivers ranged in age from 21 to 65 years with a mean of 36.35 $(S D=7.45)$. The predominant racial/ethnic identification of the women was Hispanic/Latina (60.7\%), followed by White (26.9\%), African American or Black (3.4)\%, American Indian or Alaska Native (1.7\%), Pacific Islander (0.3\%), and Asian (0.3\%); maternal caregivers who identified as more than one race comprised approximately $6.6 \%$ of the sample. Thirtysix percent of maternal caregivers did not have a high school diploma, 39\% had a high school diploma, and the remaining $25 \%$ had some college or more. The average number of years experiencing IPV for women in the sample was $9.08(S D=6.75)$. The distribution of yearly household income was: <\$9K (21.2\%), \$10K-\$29K(42.7\%), \$30K-\$49K (26.5\%), and $>\$ 50 \mathrm{~K}(9.1 \%)$. The number of additional children in the household ranged from 0 to 8 $(M=2.66, S D=1.28)$.

\section{Measures}

Children's PTS and psychopathology symptoms-Maternal caregivers completed the Child Behavior Checklist (CBCL 6/18; Achenbach \& Rescorla, 2001). The CBCL is a standardized and widely used measure of caregivers' reports of children's competencies and behavior problems with excellent psychometric properties. The measure permits calculation of raw and $T$ scores that represent multiple domains of socioemotional functioning. The present study used $T$ scores of PTS Symptoms ( $\alpha=.84)$, Internalizing Behaviors $(\alpha=.90)$, and Externalizing Behaviors ( $a=.92)$. For the PTS scale, scores between 65 and 69 reflect levels of problems that demonstrate borderline clinical significance; scores equal to or greater than 70 demonstrate clinically significant levels of problems. For the internalizing and externalizing scales, scores from 60 to 63 reflect the borderline clinical range; scores greater or equal to 64 are considered to be clinically significant levels of psychopathology symptoms.

Children's exposure to IPV—Children completed the Child Exposure to Domestic Violence Scale (CEDV; Edleson, Shin, \& Armendariz, 2008) to assess the severity of children's exposure to IPV. The CEDV measure consists of 6 subscales: (1) Violence, (2) Exposure to Violence at Home, (3) Exposure to Violence in the Community, (4) Involvement in Violence, (5) Risk Factors, and (6) Other Victimization. The 10-item "Home Exposure" 
subscale was used in the current study to assess children's exposure to IPV in the home. The CEDV asks children about different forms of IPV (e.g., violence involving weapons, injury to maternal caregiver, physical violence, emotional abuse) to which they may have been exposed and the severity of IPV exposure is indexed by summing the total number of items the child endorsed. Total severity scores can range from 0 to 10. The CEDV Home Exposure subscale includes one item about exposure to harm of animals by mothers' partners; this item was eliminated from the calculated total score due to differential item functioning across the English and Spanish translation. Therefore, the possible range of CEDV severity scores in the current study was 0 to 9 . Internal consistency for the 9-item version was sufficient $(\alpha=.80)$ and Rasch analysis confirmed the appropriateness of the 9-item version as a unidimensional construct.

Demographic information-Child- and family-level demographic variables (i.e., child gender and ethnicity, yearly household income, number of children in the household, and maternal education level) were ascertained from a demographic survey completed by the maternal caregiver. Race and ethnicity questions were asked separately and dummy variables were created to compare White Latinos, Multi-ethnic/racial, and "Other" racial minority children to White non-Latino children. Yearly household income (YHI) data were restructured so that each one-unit increase in the variable reflected a $\$ 10,000$ increase in YHI. Maternal education data was also restructured so that every one-unit increase in the variable reflected the successful completion of a grade level beyond elementary school (grade 6=0).

\section{Analytic Strategy}

Pertaining to the first research question, a regression mixture model was used to identify the optimal number of classes of participants, in which the relationships between independent variables (IPV exposure, YHI, number of children in the household) and dependent variables (PTS, internalizing, and externalizing problems) were different. Broadly speaking, regression mixture models integrate the benefits of regression models and person-centered analysis to identify differential associations between independent and dependent variables across latent subgroups using one statistical analysis (Van Horn et al., 2009; Wedel \& DeSarbo, 1995). Given that this statistical strategy is considered to be fundamentally exploratory, it was employed to examine whether a moderating factor reflecting differential effects of risk and protective factors on PTS and psychopathology symptoms could be identified in the current sample. Pearson's correlations among the study variables are provided in Table 1. The regression mixture analysis was conducted in Mplus 7.1 (Muthén \& Muthén, 2014). Models with one to four latent subgroups were tested. Residual variances for the outcomes (i.e., PTS symptoms, internalizing and externalizing problems) were allowed to be different for each class. Outcome means and the regression weights of the three symptom measures with each family contextual factor (exposure to IPV, YHI, and number of children in the household) were also allowed to differ between classes. To reduce the chance that the results were due to local maxima (see Hipp \& Bauer, 2006; Nylund, Asparauhov, \& Muthén, 2007), the number of random sets of starting values was increased to 1,000 , the number of iterations to 20 , and the number of final-stage optimizations to 100 (Asparouhov \& Muthén, 2012). 
Multiple fit indices were used to determine the best fitting model. Specifically, the Akaike information criterion (AIC), Bayesian information criterion (BIC), and adjusted BIC were examined. The best model was chosen by determining which model had the lowest values for each criterion. The bootstrapped likelihood ratio test (BLRT) was also used to determine the number of classes (Peel \& McLachlan, 2000). The BLRT tests the null hypothesis that the target model (e.g., 3 class model) fits no better than a model with one less class (e.g., 2 class model). The associated $p$-value for the BLRT denotes whether the solution with more classes $(p<.05)$ or less classes $(p>.05)$ is a better fit. In addition to fit statistics, classspecific intercepts, residual variances, and regression coefficients were examined to interpret each class (Van Horn et al., 2009). Classification certainty was also assessed using entropy values. These values can range from 0 to 1 , with higher scores representing greater classification accuracy. It is important to note, however, that Van Horn et al. (2015) argue that entropy should not be considered a class enumeration criterion when evaluating regression mixtures.

Next, the second research question was addressed by examining the moderating effects of child gender and ethnicity and maternal education. Specifically, gender and ethnicity were entered into the regression mixture model along with maternal education level as predictors of latent subgroup membership using multinomial regression.

\section{Results}

\section{Research Question 1}

The first research question examined whether or not severity of IPV exposure, YHI, and number of children in the household were differentially related to PTS and psychopathology symptoms for different children, as indicated by the presence of two or more latent classes which differed in the effects of the predictors on the outcomes. Table 2 shows the fit indices of each regression mixture solution with 1 through 4 classes. As hypothesized, results indicated evidence of a moderating factor. Specifically, multiple fit indices (i.e., AIC, BIC, adjusted BIC, and BLRT) suggested the three-class solution was the optimal number of subgroups to represent children in the sample. Furthermore, the 3-class solution demonstrated a high degree of class separation; entropy was .93 and the lowest class-specific response probability was .95 .

Next, classes were interpreted based on class-specific intercepts on the outcome variables, residual variances, and regression coefficients. Class-specific intercepts are provided in Table 3 and parameter estimates for the 3-class model are provided in Tables 4 and 5. As shown in Table 3, Class 1 comprised the largest portion of the sample (approximately 66\%; $n=190$ ) and was characterized by the lowest intercepts on all the outcome measures. This suggests that these children had relatively adaptive adjustment in the context of violent family environments. For this class, severity of exposure to IPV was significantly related to higher externalizing problems $(\beta=.20)$, but not PTS or internalizing symptoms. The number of children in the household was only significantly related to lower levels of PTS symptoms $(\beta=-.11)$. YHI was not significantly associated with any of the outcome measures. Since this group of children demonstrated relatively less psychopathological response to IPV exposure and few family contextual variables accounted for a significant amount of variance 
in PTS, internalizing, or externalizing symptoms, Class 1 was labeled as "Asymptomatic with Low Sensitivity."

Class 2 comprised approximately 24\% ( $n=69)$ of children and class-specific intercepts on the outcome measures were high, in the clinical range for internalizing and externalizing symptoms, and in the borderline clinical range for PTS symptoms. For this class, severity of IPV exposure was significantly associated with higher PTS $(\beta=.45)$ and internalizing symptoms ( $\beta=.27$ ), but not externalizing symptoms. The number of children in the household was significantly related to lower levels of PTS $(\beta=-.55)$ and internalizing symptoms ( $\beta=-.37$ ), but unrelated to externalizing symptoms. YHI was only significantly associated with externalizing symptoms $(\beta=-.37)$. This group was characterized by the strongest relationship between number of children in the family and the PTS scores. Moreover, it was the only group characterized by a statistically significant relationship between the number of other children in the household and internalizing symptoms. To reflect these relationships, this group was labeled "Maladjusted with Moderate Sensitivity." Taken together, the three family contextual variables accounted for approximately $45 \%$ of the variance in children's PTS symptoms $\left(R^{2}=.45, p<.001\right)$ and $21 \%$ of the variance in children's internalizing symptoms $\left(R^{2}=.21, p<.01\right)$.

Compared to the other subgroups, children in Class $3(10 \% ; n=30)$ had the highest intercepts on all three symptomology measures, indicating that in general, these children were characterized by significant maladjustment. All intercepts were in the clinical range. Notably, the intercept for PTS symptoms was nearly three standard deviations higher than the intercept for Class 1. For this group of children, severity of IPV exposure was significantly associated with higher levels of symptomology across all three domains (PTS: $\beta=.65$; internalizing: $\beta=.42$; externalizing: $\beta=.33$ ), and the relationships between these variables were the strongest for children in this class. In addition, YHI was significantly and negatively associated with all outcome measures (PTS: $\beta=-.62$; internalizing: $\beta=-.30$; externalizing: $\beta=-.46$ ). The relationship between YHI and outcomes was also strongest in this class. For children in Class 3, the number of children in the household was only significantly related to PTS symptoms, with the presence of more children being linked to lower PTS symptomology $(\beta=-.34)$. In light of these characteristics, this group was labeled, "Highly Maladjustment with High Sensitivity." Notably, the three family contextual variables accounted for a significant amount of variance for the PTS $\left(R^{2}=.72, p<.001\right)$ and externalizing symptoms $\left(R^{2}=.28, p<.01\right)$.

\section{Research Question 2}

Considering the high entropy of the three-class model in the context of the recommendations provided by Park, Lord, and Hart (2010), it was reasonable to continue with the next step of the analysis and use multinomial logistic regression to test the associations between gender, ethnicity, and maternal education with class membership. Results are presented in Table 5. Two covariates were significantly related to class membership: Latino ethnicity and maternal education. Specifically, results indicated that Latino children were less likely to be in Class 2 $(\mathrm{OR}=.31)$ and Class $3(\mathrm{OR}=.17)$ than Class 1 . Thus, children who were identified as Latino were more likely to be in the Asymptomatic/Low Sensitivity group than the Maladjustment/ 
Moderate Sensitivity or Highly Maladjusted/High Sensitivity groups. In addition, children of women with higher levels of education were more likely to be in the Maladjusted with Moderate Sensitivity group (Class 2) than in the Asymptomatic/Low Sensitivity group (Class 1, OR=1.02). Notably, child gender was not related to differential effects of risk and protective factors on children's PTS and psychopathology symptoms.

Van Horn et al. (2009) argue that an important question in regression mixture models is how much the interpretation of the classes changes when predictors are added. Specifically, Van Horn et al. (2009) suggest that changes in the class-specific regression weights indicate that model results are not robust. For the current study, the entropy increased from .93 in the unconditional model to .95 after the covariates were added. This indicates that these variables enhance the ability of the model to classify individuals; however this is only a slight improvement given that the precision of classification was already high. Next, the parameter estimates for each class were examined. Results indicated only a minor change in the estimates with the addition of gender, ethnicity, and maternal education. First, the relationship between severity of IPV exposure and the symptomology outcomes remained consistent across all classes. However, the association between number of children in the household and PTS symptoms changed slightly for Classes 1 and 3. Specifically, these estimates retained the same direction and relative strength; however, the statistical significance was reduced to the level of a trend $(.10>p>.05)$. Finally, the effect of YHI on internalizing symptoms in Class 3 and externalizing symptoms in Class 2 retained the direction of the effects; however, these estimates were no longer statistically significant.

\section{Discussion}

Increasingly, exposure of children to IPV is recognized as a form of child maltreatment (Gilbert et al., 2009; MacMillen et al., 2013). Using the regression mixture model approach, results of this study provide empirical support for differential effects of IPV exposure severity and family environmental factors on children's adjustment outcomes in the context of IPV. Specifically, the present study demonstrated three major findings. First, this study identified three distinct latent groups of children who differed in PTS symptoms and internalizing and externalizing symptoms, and the relationships of those outcomes with severity of IPV exposure, YHI, and the number of children in the household. They include the asymptomatic with low sensitivity to environmental factors (Class 1), the maladjusted with moderate sensitivity (Class 2 ) and the highly maladjusted with high sensitivity (Class 3). Second, compared to White non-Latino children, Latino children were significantly less likely to be in the maladjusted (Class 2) and highly maladjusted (Class 3) classes. Finally, children with high levels of maternal education were more likely to be in the maladjusted with moderate sensitivity class (Class 2 ) than the asymptomatic with low sensitivity class (Class 1).

The asymptomatic class with low sensitivity to environmental factors (Class 1; 66\%) included children whose symptomatology outcomes were generally not related to the family contextual factors such as YHI and number of children in the household. However, there was an association between IPV exposure severity and externalizing problems that remained statistically significant after the differential effects predictors were added to the model. Next, 
for the maladjusted with moderate sensitivity class (Class 2; 24\%), IPV exposure severity was significantly related to increased levels of PTS symptoms and internalizing problems, but not externalizing problems. Furthermore, the number of children in the household was significantly and negatively related to both PTS symptoms and internalizing problems. Finally, children in the highly maladjusted group with high environmental sensitivity (Class 3 ) had the most severe levels of adjustment problems. This group made up about $10 \%$ of the sample and was distinguished by statistically significant relationships between IPV exposure severity and YHI with all PTS and psychopathology symptoms. It is interesting to note that the highly maladjustment group was distinguished by having the highest internalizing problems intercept; however, the family contextual factors examined in the current study did not explain a significant amount of variance in internalizing problem scores. Notably, the effects of IPV exposure on the outcome measures were the most helpful variable in describing and distinguishing between these classes. Taken as a whole, these results suggest that IPV exposure severity and family context factors are more strongly associated to PTS and psychopathology symptoms among the maladjusted groups (i.e., Class 2 and 3); YHI is related to the adjustment outcomes only among maladjusted groups; and the number of kids at home was consistently related to PTS symptoms across latent groups

Prior variable-centered research has demonstrated that some children have a more internalizing trajectory following exposure to IPV and other children are characterized by a more externalizing trajectory (Graham-Bermann \& Levendosky, 2011). Furthermore, some children demonstrate patterns of adjustment suggesting that they are resilient to the impact of IPV, while others experience compromised functioning across multiple domains (Graham-Bermann et al., 2009). The results of the present study lend support to these prior findings and provide evidence in support of the hypothesis that groups of children can be distinguished based on differential effects of IPV exposure severity on child outcomes. Identification of the latent moderating factor provides a strong argument for the use of person-centered methods in future research to examine psychosocial outcomes among children exposed to family violence. Through the identification of latent groups, this study was able to document a large portion of resilient children with low sensitivity to the impact of IPV exposure severity and family context factors on psychopathology. In addition, two smaller subgroups that were characterized by potentially internalizing vs. comorbid psychosocial trajectories emerged from the analysis. Results surrounding the large asymptomatic group should be interpreted with caution, however, as no strength-based outcomes (e.g., competence, self-worth) were examined. Prior research (Graham-Bermman et al., 2009) has suggested that such measures are important in distinguishing resilient children from those with asymptomatic profiles of functioning.

Differential effects for YHI and number of children in the household were also identified. While YHI was negatively correlated with all outcomes in the highly maladjusted with high environmental sensitivity class and externalizing problems in the maladjusted with moderate sensitivity class, there was no relationship between YHI and outcomes for the asymptomatic with low sensitivity class. This finding suggests that YHI may serve as a buffering agent for PTS and psychopathology symptoms particularly in maladjusted child victims of IPV. Graham-Bermann et al. (2006) note that YHI may buffer the impact of IPV exposure on children's PTS symptoms by increasing vulnerable families' access to other resources. 
Results of the current study also demonstrated that the number of children in the household was consistently negatively associated with PTS symptoms for each latent group and with internalizing problems for the maladjusted with moderate sensitivity class. Carlson and Corcoran (2001) suggest that as the number of children in households increases, mothers may be less likely to be aware of their child's psychosocial difficulties, possibly due to compromised parenting resulting from increased stress and less financial stability. However, our findings suggest that even among mothers who noted high levels of psychosocial difficulties for their child, the number of siblings in the household was negatively associated with trauma symptomology. Thus, as argued by several researchers, siblings may help to augment socioemotional development and diffuse the effects of negative family factors (Steelman, Powell, Werum, \& Carter, 2002: Downey \& Condron, 2004). Given that many studies have also found that larger families with more children in the household are at increased risk for violence and compromised child developmental outcomes (Hoffman et al., 1994; Keenan et al., 2007; Trentacosta et al., 2008), more research is needed on the influence of the number of children in the household as it pertains to children's adjustment in the context of IPV.

After finding evidence for differential risk and protective effects, the present study examined the effects of child gender, ethnicity, and maternal education on the class memberships. Consistent with prior person-centered research, gender was not significantly related to differential effects (e.g., Hughes \& Luke, 1998; Lang \& Stover, 2008). Regarding ethnicity, only Latino ethnicity was related to class membership. Specifically, Latino children were significantly less likely to be in the maladjusted and highly maladjusted groups. This finding is not surprising given evidence that children from Latino families, even when faced with cumulative risks such as low socioeconomic, low maternal education, and IPV, experience fewer socioemotional and behavioral problems than their White European American peers (Ansari \& Winsler, 2012; Crosnoe, 2006; De Feyter \& Winsler, 2009; McDonald et al., in press). Lastly, our results also indicated that children with mothers who had higher levels of education were more likely to be in the maladjusted/moderate sensitivity class than the asymptomatic/low sensitivity group. Given that this class (Class 2) was distinguished by moderate sensitivity to IPV for PTS and internalizing problems only, this result is somewhat consistent with Hughes and Luke's (1998) findings, which reported that children with predominantly depressed patterns of adjustment had mothers who were more educated than children with other patterns of adjustment. Since there are mixed findings with respect to the effect of maternal education on child psychopathology in the context of IPV, future longitudinal studies are warranted to explore how maternal education influences the onset and course of child psychopathology trajectories among families exposed to IPV.

\section{Limitations}

A limitation of the current study concerns the size of the sample. Regression mixture modeling is a large sample technique and there is conflicting evidence regarding the implementation of this technique in small samples (i.e., $\mathrm{N}<300$ ). Due to the restricted sample size, only a small number of family context factors could be examined. Incorporation of a great number of risk and protective factors would provide a more holistic ecological model of differential effects among children exposed to family violence. For example, 
children's exposure to additional traumatic events (e.g., physical abuse) beyond exposure to IPV might have been influential. Similarly, a measure that weights different forms of IPV in terms of their severity and duration would be an improvement, as the CEDV score used in the present study relies on the number of items endorsed, valuing emotional abuse the same as acts of severe physical violence. Furthermore, many families experiencing IPV do not seek out community-based services and may not have pets living in the home, which were the inclusion criteria for the present study. Therefore, results of this study may not generalize to families in the larger community who are impacted by IPV or those without pets. Moreover, due the sample composition and small proportion of children in Class 3, many racial and ethnic differences could not be explored in a meaningful way. It is also important to note that the large percentage of asymptomatic children could be attributed to the fact that women who had more than one child in the 7 to 12 age range were able to select which child participated in the study; mothers may have selected the child with the least amount of behavioral challenges who was more likely to successfully complete the survey materials. Another limitation concerns the use of cross-sectional data, which makes it difficult to consider causal relationships between IPV exposure and psychopathological symptoms. This study is also limited by the manner in which family income and the number of children in the household were measured. Incorporation of a more dynamic measure of socioeconomic status, such as a poverty ratio, may have provided better differentiation between the classes. Furthermore, while the number of children in the household sheds some light on the impact of family size on child outcomes in the context of IPV, examining the quality of sibling relationships and/or the total number of people living in the household would have provided knowledge with greater implications for practice and intervention efforts. This study was also unable to collect data on maternal warmth and parenting due to considerations related to survey length, participant burden, and response quality. These parenting factors are important variables to consider when examining the impact of IPV on children (Graham-Bermann et al., 2009) and may have assisted in distinguishing between the differential effects classes. Finally, this study only examined constructs reflecting psychopathology and did not incorporate other measures of resilient functioning such as developmental competence.

\section{Implications for research and practice}

It is widely accepted that understanding patterns of risk and protection among children exposed to family violence can help guide effective and tailored interventions. However, the majority of analytic approaches employed in this area of study assume comparable effects of contextual risk and protective factors for all children. Prior person-centered studies have provided evidence of heterogeneity of outcomes among children exposed to IPV, and the need to develop effective interventions that are designed to address specific patterns of adjustment rather than the traditional "one-size-fits-all" approach (Graham-Bermann, Miller-Graff, Howell \& Grogan-Kaylor, 2015). Findings from the current study lend support to this argument, and provide additional information suggesting that heterogeneity among children exposed to IPV extends to differential effects of risk and protective factors as well. Clearly, children in the Classes 2 and 3 are in need of services to address their adjustment problems and high levels of psychopathology. Less clear are the needs of children in the largest group (Class 1). While not all children exposed to IPV are in the clinical range of 
psychopathology, many in this group were not free of symptoms altogether, suggesting that attention be paid to determining who among them might be most at-risk. The finding that children in Class 1 who were exposed to more severe IPV had higher externalizing problems implies the need for screening all children who have exposure to IPV to determine their needs, even though they demonstrate few symptoms of psychopathology.

Through regression mixture models, differential effects can be found in an empirical manner; however, Van Horn et al. $(2009,2015)$ warn that this approach is largely data driven. Therefore, it is critical that the results of studies are replicated in other samples to explore whether different samples yield different results. Given the limitation of the crosssectional design described above, future research should explore if there are latent subgroups of children defined by differential effects of IPV on long-term outcomes. Differential effects of exposure to direct forms of child abuse and neglect should also be explored, independently and in combination with IPV exposure. Identifying predictors of differential effects using longitudinal designs could identify important intervention targets that might promote resilience across time.

As a whole, this study adds to the growing body of literature suggesting that intervention efforts for children exposed to IPV must account for diverging trajectories of internalizingspecific, externalizing-specific, and co-morbid symptomology (Graham-Bermann et al., 2009; Spilsbury et al., 2008). Additional studies incorporating larger, more diverse samples are needed to examine individual differences in the effects of IPV exposure and related risk and protective factors on child adjustment outcomes. Furthermore, it is essential that future studies of differential effects incorporate variables that can be targeted by intervention and prevention programs as this may help to bolster intervention efforts with families impacted by IPV.

\section{Acknowledgments}

This research was funded by Grant 5R01-HD-66503-4 from the Eunice Kennedy Shriver National Institute of Child Health and Human Development (NICHD) and Grant 2015-0709 from the American Society for the Prevention of Cruelty to Animals (ASPCA). The content is solely the responsibility of the authors and does not necessarily represent the official views of the National Institutes of Health or ASPCA. The authors would like to thank the community-based domestic violence advocates for their contribution to this work.

\section{References}

Achenbach, T.; Rescorla, L. Manual for the ASEBA school-age forms \& profiles. Burlington, VT: University of Vermont, Research Center for Children, Youth, \& Families; 2001.

Ansari A, Winsler A. School readiness among low-income, Latino children attending family childcare versus centre-based care. Early Child Development and Care. 2012; 182(11):1465-1485.

Asparouhov T, Muthén B. Using Mplus tech11 and tech14 to test the number of latent classes. 2012 (Mplus Web Notes No. 14). Retrieved from http://www.statmodel.com/examples/webnotes/ webnote14.pdf.

Bergman LR, Magnusson D. A person-oriented approach in research on developmental psychopathology. Development and Psychopathology. 1997; 9:291-319. [PubMed: 9201446]

Bergman LR, Trost K. The person-oriented versus the variable-oriented approach: Are they complementary, opposites, or exploring different worlds? Merrill-Palmer Quarterly. 2006; 52(3): 601-632. 
Bronfenbrenner, U. The ecology of human development: Experiments in nature and design. Cambridge, MA: Harvard University Press; 1979.

Bronfenbrenner, U. Making human beings human: Bioecological perspectives on human development. Thousand Oaks, CA: Sage Publications; 2005.

Carlson MJ, Corcoran ME. Family structure and children's behavioral and cognitive outcomes. Journal of Marriage and Family. 2001; 63(3):779-792.

Chan Y, Yeung J. Children living with violence within the family and its sequel: A meta-analysis from 1995-2006. Aggression and Violent Behavior. 2009; 14(5):313-322.

Crosnoe, R. Mexican roots, American schools: Helping Mexican immigrant children succeed. Stanford, CA: Stanford University Press; 2006.

De Feyter JJ, Winsler A. The early developmental competencies and school readiness of low-income, immigrant children: Influences of generation, race/ethnicity, and national origins. Early Childhood Research Quarterly. 2009; 24(4):411-431.

Ding C. Using regression mixture analysis in educational research. Practical Assessment Research and Evaluation. 2006; 11(11)

DiStefano C, Kamphaus RW. Investigating subtypes of child development: A comparison of cluster analysis and latent class cluster analysis in typology creation. Educational and Psychological Measurement. 2006; 66:778-794.

Downey DB, Condron DJ. Playing well with others in kindergarten: The benefit of siblings at home. Journal of Marriage and Family. 2004; 66(2):333-350.

Edleson, J. Should childhood exposure to adult domestic violence be defined as child maltreatment under the law?. In: Jaffe, P.; Baker, L.; Cunningham, A., editors. Protecting Children From Domestic Violence: Strategies for Community Intervention. New York, NY: Guilford Press; 2004. p. 8-29.

Edleson JL, Shin N, Armendariz KKJ. Measuring children's exposure to domestic violence: The development and testing of the Child Exposure to Domestic Violence (CEDV) Scale. Children and Youth Services Review. 2008; 30:502-521.

Evans SE, Davies C, DiLillo D. Exposure to domestic violence: A meta-analysis of child and adolescent outcomes. Aggression and Violent Behavior. 2008; 13:131-140.

Foster H, Brooks-Gunn J. Toward a stress process model of children's exposure to physical family and community violence. Clinical Child and Family Psychology Review. 2009; 12:71-94. [PubMed: 19434492]

Gewirtz AH, Edleson JL. Young children's exposure to intimate partner violence: Towards a developmental risk and resilience framework for research and intervention. Journal of Family Violence. 2007; 22:151-163.

Graham-Bermann SA, DeVoe ER, Mattis JS, Lynch S, Thomas SA. Ecological predictors of traumatic stress symptoms in Caucasian and ethnic minority children exposed to intimate partner violence. Violence Against Women. 2006; 12(7):662-692.

Graham-Bermann SA, Gruber G, Howell KH, Girz L. Factors discriminating among profiles of resilient coping and psychopathology in children exposed to domestic violence. Child Abuse \& Neglect. 2009; 33(9):648-660. [PubMed: 19804905]

Graham-Bermann SA, Levendosky AA. The social functioning of preschool-age children whose mothers are emotionally and physically abused. Journal of Emotional Abuse. 1998; 1(1):59-84.

Graham-Bermann, SA.; Levendosky, AA. Introduction. In: Graham-Bermann, SA.; Edleson, JL., editors. Domestic violence in the lives of children: The future of research, intervention, and social policy. Washington, DC: American Psychological Association; 2011. p. 13-34.

Graham-Bermann SA, Miller-Graff LE, Howell KH, Grogan-Kaylor A. An efficacy trial of an intervention program for young children exposed to intimate partner violence. Child Psychiatry \& Human Development. 2015 Advance online publication.

Grych JH, Jouriles EN, Swank PR, McDonald R, Norwood WD. Patterns of adjustment among children of battered women. Journal of Consulting and Clinical Psychology. 2000; 68:84-94. [PubMed: 10710843]

Hamby, SL.; Finkelhor, D.; Turner, HA.; Ormrod, RK. Children's exposure to intimate partner violence and other forms of family violence: Nationally representative rates among US youth. 
Washington, DC: US Government Printing Office; 2011. OJJDP Juvenile Justice Bulletin - NCJ 232272

Hipp JR, Bauer DJ. Local solutions in the estimation of growth mixture models. Psychological Methods. 2006; 11(1):36. [PubMed: 16594766]

Hoffman K, Demo D, Edwards J. Physical wife abuse in a non-Western society: An integrated theoretical approach. Journal of Marriage and the Family. 1994; 56:131-146.

Holt S, Buckley H, Whelan S. The impact of exposure to domestic violence on children and young people: A review of the literature. Child Abuse and Neglect. 2008; 32:797-810. [PubMed: 18752848]

Howell KH, Graham-Bermann SA, Czyz E, Lilly M. Assessing resilience in preschool-age children exposed to intimate partner violence. Violence \& Victims. 2010; 25(2):150-164. [PubMed: 20514814]

Howell KH, Barnes SE, Miller LE, Graham-Bermann SA. Developmental variations in the impact of intimate partner violence exposure during childhood. Journal of Injury and Violence Research. $2015 ; 8(1)$ in press.

Hughes, HM.; Luke, DA. Heterogeneity in adjustment among children of battered women. In: Holden, GW.; Geffner, R.; Jouriles, EN., editors. Children exposed to marital violence: Theory, research, and applied issues. Washington, DC: American Psychological Association; 1998. p. 185-221.

Gilbert R, Widom CS, Browne K, Fergusson D, Webb E, Janson S. Burden and consequences of child maltreatment in high-income countries. Lancet. 2009; 373(9657):68-81. [PubMed: 19056114]

Keenan K, Gunthorpe D, Grace D. Parsing the relations between SES and stress reactivity: Examining individual differences in neonatal stress response. Infant Behavior and Development. 2007; 30(1): 134-145. [PubMed: 17292786]

Kilpatrick KL, Williams LM. Potential mediators of post-traumatic stress disorder in child witnesses to domestic violence. Child Abuse \& Neglect. 1998; 22(4):319-330. [PubMed: 9589182]

Kitzmann K, Gaylord N, Holt A, Kenny E. Child witness to domestic violence: A meta-analytic review. Journal of Consulting and Clinical Psychology. 2003; 71:339-352. [PubMed: 12699028]

Kuelbs, CL. The impact of intimate partner violence on children. In: Mitchell, C.; Anglin, D., editors. Intimate partner violence: A health-based perspective. New York, NY: Oxford University Press; 2009. p. 499-509.

Lang JM, Stover CS. Symptom patterns of youth exposed to intimate partner violence. Journal of Family Violence. 2008; 23:619-629.

MacMillan HL, Wathen CN, Varcoe CM. Intimate partner violence in the family: Considerations for children's safety. Child Abuse \& Neglect. 2013; 37(12):1186-1191. [PubMed: 23830555]

McDonald SE, Graham-Bermann SA, Maternick A, Ascione F, Williams JH. Patterns of Adjustment among Children Exposed to Intimate Partner Violence: A Person-Centered Approach. Journal of Child \& Adolescent Trauma. (in press).

Muthén BO, Asparouhov T. Multilevel regression mixture analysis. Journal of the Royal Statistical Society. 2009; 172:639-657.

Muthén, L.; Muthén, B. Mplus user's guide: Statistical analysis with latent variables. Seventh. Los Angeles, CA: Muthén \& Muthén; 2014.

Nylund KL, Asparouhov T, Muthén BO. Deciding on the number of classes in latent class analysis and growth mixture modeling: A Monte Carlo simulation study. Structural Equation Modeling. 2007; 14(4):535-569.

Park BJ, Lord D, Hart JD. Bias properties of Bayesian statistics in finite mixture of negative binomial regression models in crash data analysis. Accident Analysis \& Prevention. 2010; 42(2):741-749. [PubMed: 20159102]

Peel D, McLachlan GJ. Robust mixture modeling using the t-distribution. Statistics \& Computing. 2000; 10(4):339-348.

Shaw DS, Keenan K, Vondra JI, Delliquandri E, Giovannelli J. Antecedents of preschool children's internalizing problems: A longitudinal study of low-income families. Journal of the American Academy of Child and Adolescent Psychiatry. 1997; 36:1760-1767. [PubMed: 9401338] 
Skopp NA, McDonald R, Jouriles EN, Rosenfield D. Partner aggression and children's externalizing problems: Maternal and partner warmth as protective factors. Journal of Family Psychology. 2007; 21:459-467. [PubMed: 17874931]

Spilsbury JC, Kahana S, Drotar D, Creeden R, Flannery DJ, Friedman S. Profiles of behavioral problems in children who witness domestic violence. Violence and Victims. 2008; 23(1):3-17. [PubMed: 18396578]

Steelman LC, Powell B, Werum R, Carter S. Reconsidering the effects of sibling configuration: Recent advances and challenges. Annual Review of Sociology. 2002; 28:243-269.

Sternberg KJ, Baradaran LP, Abbott CB, Lamb ME, Guterman E. Type of violence, age, and gender differences in the effects of family violence on children's behavior problems: A mega-analysis. Developmental Review. 2006; 26(1):89-112.

Trentacosta CJ, Hyde LW, Shaw DS, Dishion TJ, Gardner F, Wilson M. The relations among cumulative risk, parenting, and behavior problems during early childhood. Journal of Child Psychology \& Psychiatry. 2008; 49(11):1211-1219. [PubMed: 18665880]

Van Horn ML, Jaki T, Masyn K, Howe G, Feaster DJ, Lamont AE, Kim M. Evaluating differential effects using regression interactions and regression mixture models. Educational and Psychological Measurement. 2015; 75:677-714. [PubMed: 26556903]

Van Horn ML, Jaki T, Masyn K, Ramey SL, Smith JA, Antaramian S. Assessing differential effects: Applying regression mixture models to identify variations in the influence of family resources on academic achievement. Developmental Psychology. 2009; 45:1298-1313. [PubMed: 19702393]

von Eye A, Bogat GA. Person-oriented and variable-oriented research: Concepts, results, and development. Merrill-Palmer Quarterly. 2006; 52(3):390-420.

Wedel M, DeSarbo WS. A mixture likelihood approach for generalized linear models. Journal of Classification. 1995; 12(1):21-55.

Wolfe DA, Crooks CV, Lee V, McIntyre-Smith A, Jaffe PG. The effects of children's exposure to domestic violence: A meta-analysis and critique. Clinical Child and Family Psychology Review. 2003; 6:171-187. [PubMed: 14620578] 


\section{롤 \\ 골}

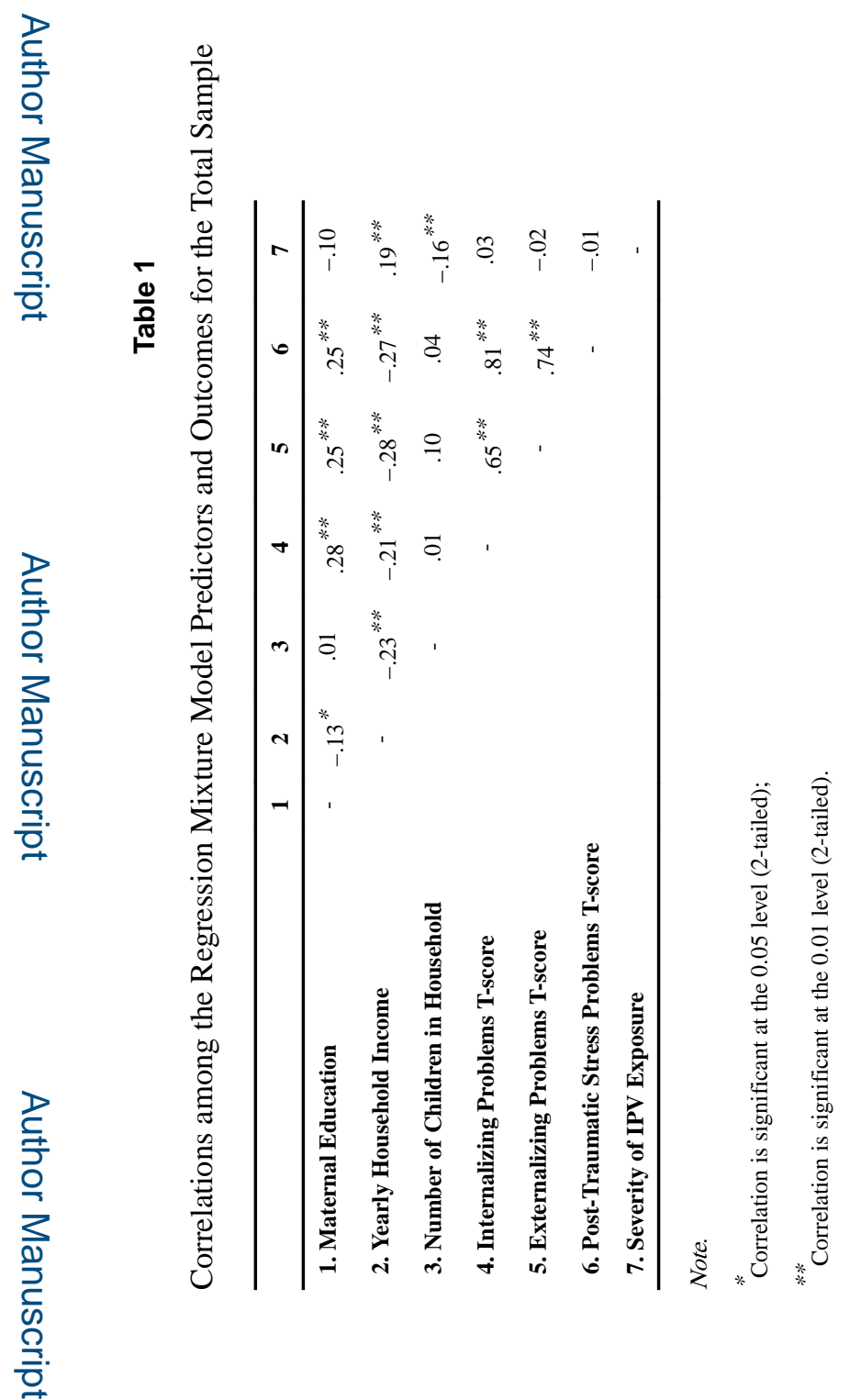

Child Abuse Negl. Author manuscript; available in PMC 2017 August 01. 


\section{로을}

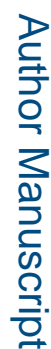

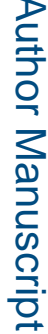

$\frac{\text { }}{\frac{0}{\circ}}$

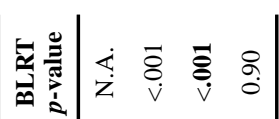

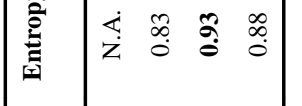

莡

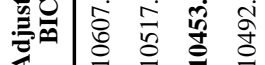
$\fallingdotseq \stackrel{\circ}{\circ} \infty$ 
Table 3

Class Size and Class- Specific Intercepts on the Outcomes for the 3-class Model

\begin{tabular}{lcccc}
\hline & $\boldsymbol{n}$ & $\begin{array}{c}\text { PTS } \\
\text { Symptoms }\end{array}$ & $\begin{array}{c}\text { INT } \\
\text { Problems }\end{array}$ & $\begin{array}{c}\text { EXT } \\
\text { Problems }\end{array}$ \\
\hline Sample & 289 & 61.31 & 58.31 & 55.23 \\
3 class solution & & & & \\
Class 1-(66\%) & 190 & 51.65 & 47.79 & 43.24 \\
Class 2-(24\%) & 69 & 67.12 & 67.67 & 69.07 \\
Class 3-(10\%) & 30 & 81.37 & 75.44 & 69.78 \\
\hline
\end{tabular}

Note. PTS= Posttraumatic Stress; INT= Internalizing; EXT= Externalizing 


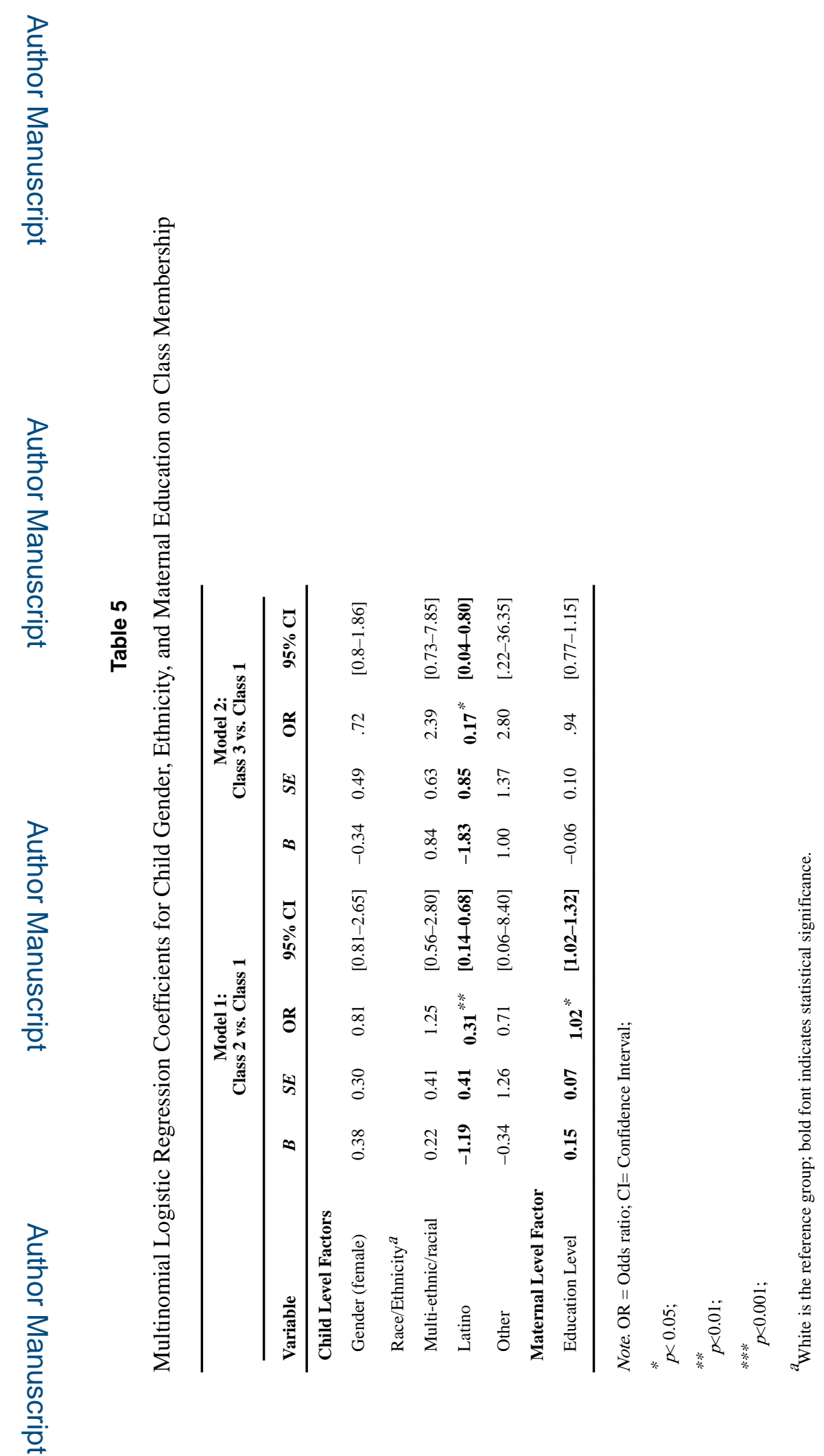

Child Abuse Negl. Author manuscript; available in PMC 2017 August 01. 